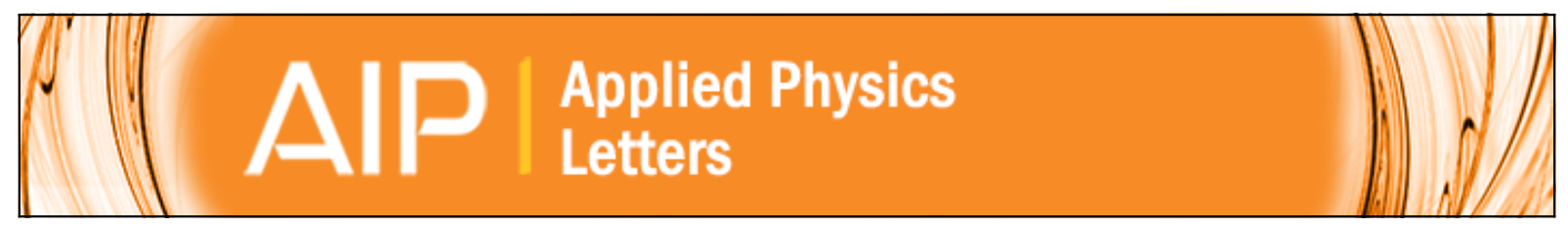

\title{
Soft molding lithography of conjugated polymers
}

Dario Pisignano, Luana Persano, Roberto Cingolani, Giuseppe Gigli, Francesco Babudri, Gianluca M. Farinola, and Francesco Naso

Citation: Applied Physics Letters 84, 1365 (2004); doi: 10.1063/1.1644921

View online: http://dx.doi.org/10.1063/1.1644921

View Table of Contents: http://scitation.aip.org/content/aip/journal/apl/84/8?ver=pdfcov

Published by the AIP Publishing

\section{Articles you may be interested in}

Rigid organic molds for nanoimprint lithography by replica molding of high glass transition temperature polymers J. Vac. Sci. Technol. B 22, 1759 (2004); 10.1116/1.1767108

Embossing of polymers using a thermosetting polymer mold made by soft lithography J. Vac. Sci. Technol. B 21, 1318 (2003); 10.1116/1.1585066

Reversal imprinting by transferring polymer from mold to substrate J. Vac. Sci. Technol. B 20, 2872 (2002); 10.1116/1.1523404

Three-dimensional pattern transfer and nanolithography: modified soft molding Appl. Phys. Lett. 81, 1011 (2002); 10.1063/1.1498149

Fabrication of three-dimensional microstructures by soft molding Appl. Phys. Lett. 79, 2285 (2001); 10.1063/1.1407859 


\title{
Soft molding lithography of conjugated polymers
}

\author{
Dario Pisignano, a) Luana Persano, Roberto Cingolani, and Giuseppe Gigli \\ National Nanotechnology Laboratory, Istituto Nazionale di Fisica della Materia (INFM), c/o Dipartimento \\ di Ingegneria dell'Innovazione, Università di Lecce, Via Arnesano, I-73100 Lecce, Italy
}

Francesco Babudri, Gianluca M. Farinola, and Francesco Naso

CNR ICCOM, Sezione di Bari and Dipartimento di Chimica, Università di Bari, via Amendola 173, I-70126 Bari, Italy

(Received 3 April 2003; accepted 3 December 2003)

\begin{abstract}
We report on the nanopatterning of conjugated polymers by soft molding, and exploit the glass transition of the organic compound in conformal contact with an elastomeric element. We succeeded in printing different compounds with resolution down to $300 \mathrm{~nm}$ at temperatures up to $300{ }^{\circ} \mathrm{C}$ in vacuum. No significant variation of the photoluminescence (PL) spectra nor heavy degradation of the PL quantum yield was observed after the lithography process. Based on the high resolution achieved and on the well-retained luminescence properties of the patterned compounds, we conclude that high-temperature soft lithography is a valid, flexible and straightforward technique for one-step realization of organic-based devices. (C) 2004 American Institute of Physics.
\end{abstract}

[DOI: 10.1063/1.1644921]

Soft lithographies ${ }^{1}$ represent a very appealing route to micro- and nanofabrication because of their high resolution, very wide chemical flexibility, large area operation, and low cost. They are based on the conformal contact between the material to be patterned and an elastomeric replica of a master structure. The most recent and promising method is soft molding (SM), or capillary force lithography, ${ }^{2}$ which combines soft and nanoimprint lithography, ${ }^{3}$ using elastomeric elements and exploits the glass transition of organic compounds. In SM lithography the mold (in this case elastomeric) is placed onto a polymeric film, which is driven above the glass transition temperature, $T_{g}$, of the target compound. Subsequent cool down below $T_{g}$ freezes the pattern into the polymer, and the replica can be peeled off.

SM allows one to overcome the main limitations of nanoimprint lithography. In fact, since penetration of the polymer into recessed features of the replica is driven by capillarity effects, SM is only marginally affected by problems of difficult polymer transport. Moreover, SM is cheaper since it does not need any pressing setup to ensure contact between the mold and the polymer. SM can also be used as a low-cost alternative to traditional nanofabrication techniques on inorganic surfaces, because the imprinted pattern can be transferred to the substrate by an $\mathrm{O}_{2}$ plasma process onto resists (to remove the eventual polymer bottom layer) followed by reactive ion etching (RIE) of the substrate. ${ }^{4}$ Finally, SM does not present pattern shrinkage and distortion due to the solvents employed by other soft lithography techniques. ${ }^{5}$ Notwithstanding these results, to date SM has been applied only to a limited class of materials. ${ }^{2,4,6}$

In this letter, we demonstrate that SM can be successfully employed also for direct patterning of optically functional molecules, thereby opening the way for one-step realization of organic-based devices. We succeeded in directly

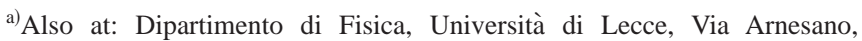
I-73100 Lecce, Italy; electronic mail: dario.pisignano@unile.it
}

printing different conjugated polymers with resolution down to $300 \mathrm{~nm}$, without severe degradation of the emission properties.

A schematic of the lithography process is shown in Fig. 1(a). First, we fabricated our master structures onto $\mathrm{Si}$ by both photo- and electron-beam lithography using a Raith Elphy Plus pattern generator, and subsequent RIE by a $\mathrm{CF}_{4}$ /Ar mixture. We realized gratings with periods of 600 $\mathrm{nm}$, which can be employed as templates for the fabrication
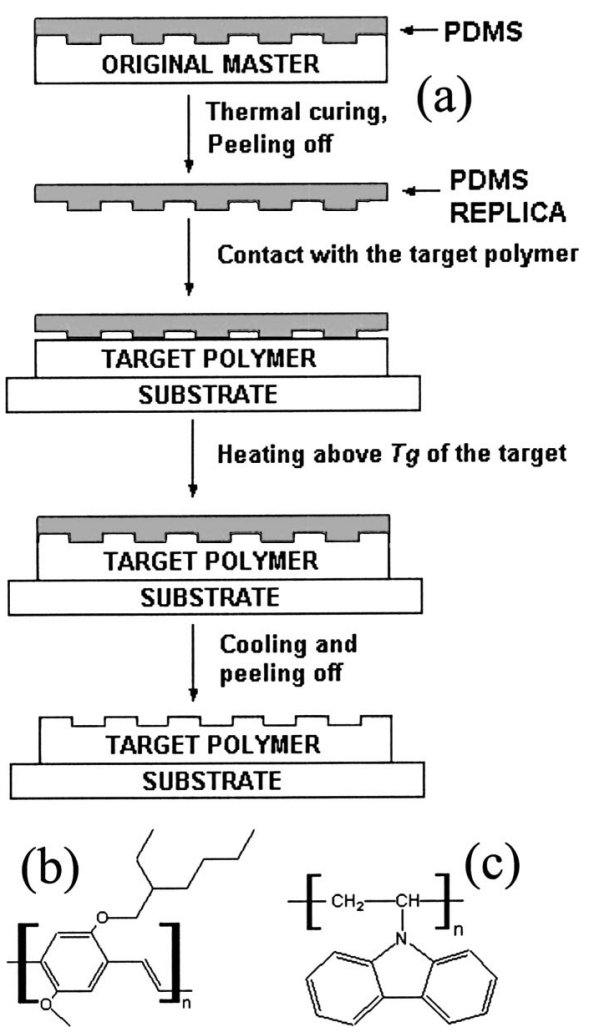

FIG. 1. (a) Schematic diagram of the process of master replication and soft molding (features not to scale). Bottom: Molecular structures of MEH-PPV (b) and PVK (c). 


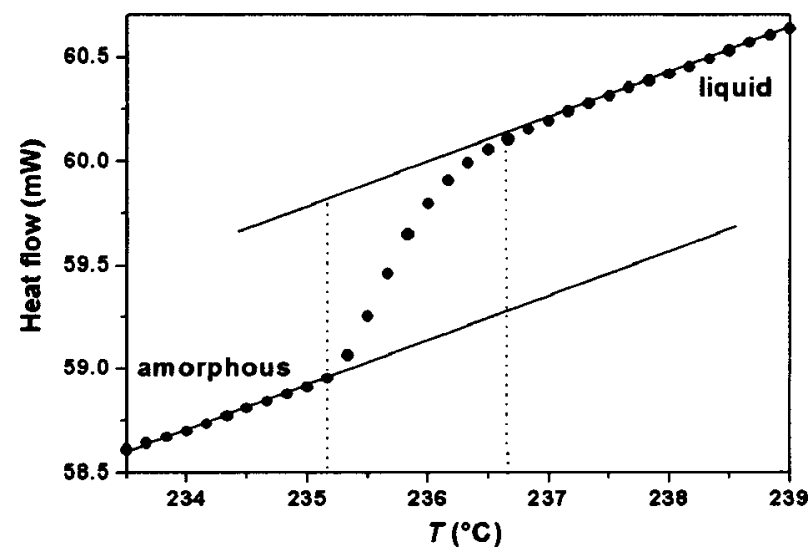

FIG. 2. Differential scanning calorimetry trace for PVK (with a heating rate of $10{ }^{\circ} \mathrm{C} / \mathrm{min}$ under nitrogen protection). The solid lines are guides for the eye; the dotted lines indicate the glass transition region. $T_{g}$ is at about $236^{\circ} \mathrm{C}$.

of distributed feedback cavities. Elastomeric replicas of the master were realized by poly(dimethylsiloxane) (PDMS) (Sylgard 184, Dow Corning, Midland, MI) according to a standard replica molding procedure, ${ }^{1}$ and placed onto spincast polymer films under their own weight. SM was carried out on different prototype light-emitting polymers [the molecular structures in Figs. 1(b) and 1(c)], which are known to exhibit good PL performance and are used to fabricate solid state lasers ${ }^{7}$ and light emitting devices: ${ }^{8,9}$ poly[2 - methoxy - 5 - (2' - ethylhexiloxy)-1,4-phenylenevinylene] (MEHPPV) and poly(9-vinylcarbazole) (PVK). The influence of the lithography procedure on the PL yield was tested by measuring the PL absolute quantum efficiency, $\Phi$, of the polymer films by placing the samples into an integrating sphere ${ }^{10}$ before and after the patterning.

Since oxygen is known to be responsible for heavy luminescence quenching in organic molecules, particularly through the oxidation of the vinylene units in poly(phenylenevinylene) compounds, ${ }^{11}$ our SM procedures were performed on a hot plate in a high-vacuum chamber $\left(10^{-5}\right.$ mbar) to prevent degradation of the molecular properties during the thermal cycle. ${ }^{12}$ The $T_{g}$ of our compounds is between 210 (Ref. 8) and $240^{\circ} \mathrm{C}$ [an example of a differential scanning calorimetry (DSC) trace is shown in Fig. 2]. The imprinting processes were carried out at a temperature up to about $300^{\circ} \mathrm{C}$. The master pattern and its transfer to MEH-PPV are shown in Figs. 3(a) and 3(b), respectively. Unlike nanoimprint lithography, SM uses intermediate replicas to transfer the pattern thus preserving the same shape (positive) of the master. The high fidelity of the pattern and the large patterned area, even with features as low as $300 \mathrm{~nm}$, can be clearly observed from a comparison of Figs. 3(a) and 3(b). The periodicity obtained faithfully reproduces that of the master, and the two-dimensional view and cross section of the final polymer gratings, imaged by atomic force microscopy (AFM) in Fig. 4, show a welldefined pattern, whose high quality suggests that the resolution achievable can be further reduced, depending on the starting masters.

SM is based on a capillarity effect that forces the polymer to penetrate into the recessed features of PDMS. Such penetration is favored by a low polymer viscosity, since the
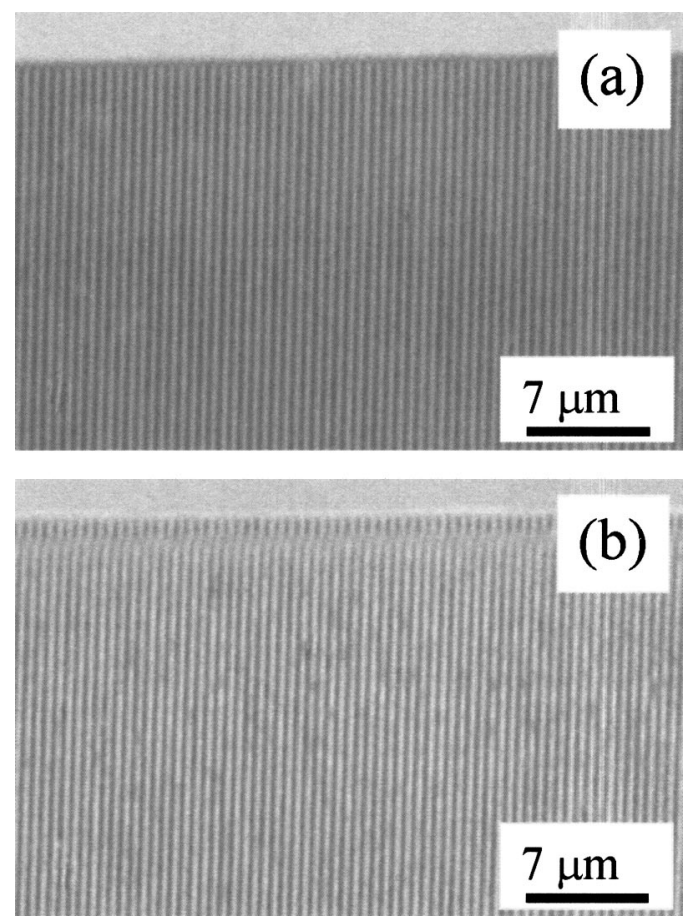

FIG. 3. Optical micrographs of master structures (a) and SM results (b) on MEH-PPV.

time, $t$, needed for the filling process is proportional to the square of the feature height, $z$, and to the polymer viscosity, $\eta:^{13}$

$$
t=\frac{2 \eta z^{2}}{\gamma R \cos \Theta},
$$

where $\gamma, R$, and $\Theta$ indicate the fluid-air surface tension, the hydraulic radius of the capillary, and the contact angle between the liquid and the surface of the capillary, respectively. In many organic compounds the viscosity above the glass transition depends on temperature according to ${ }^{14}$

$$
\eta(T)=\eta_{0} \exp \left(\frac{D T_{0}}{T-T_{0}}\right),
$$

where $D$ is referred to as the strength parameter and $T_{0}$ is the temperature for which Eq. (2) shows a divergence in polymer

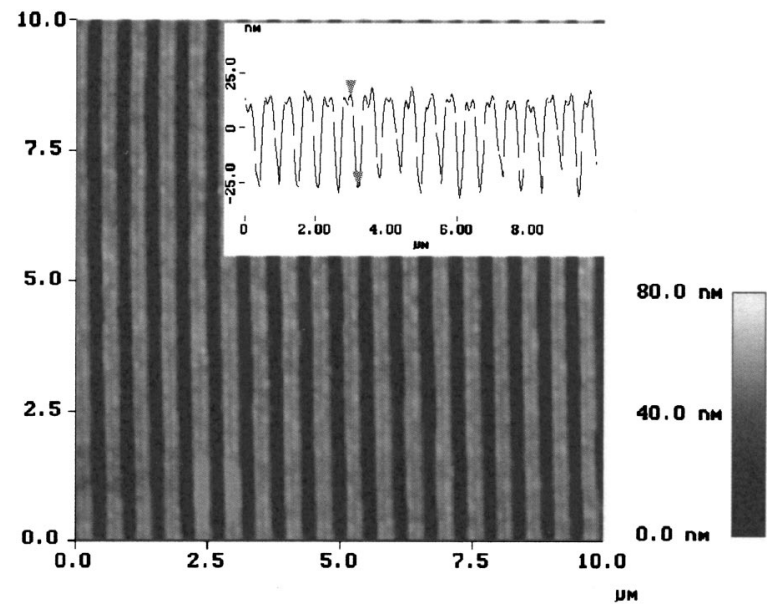

FIG. 4. AFM two-dimensional view of a patterned polymer grating (10 $\times 10 \mathrm{\mu m}^{2}$ ) and corresponding cross section (inset). The height of the features is about $40 \mathrm{~nm}$. 

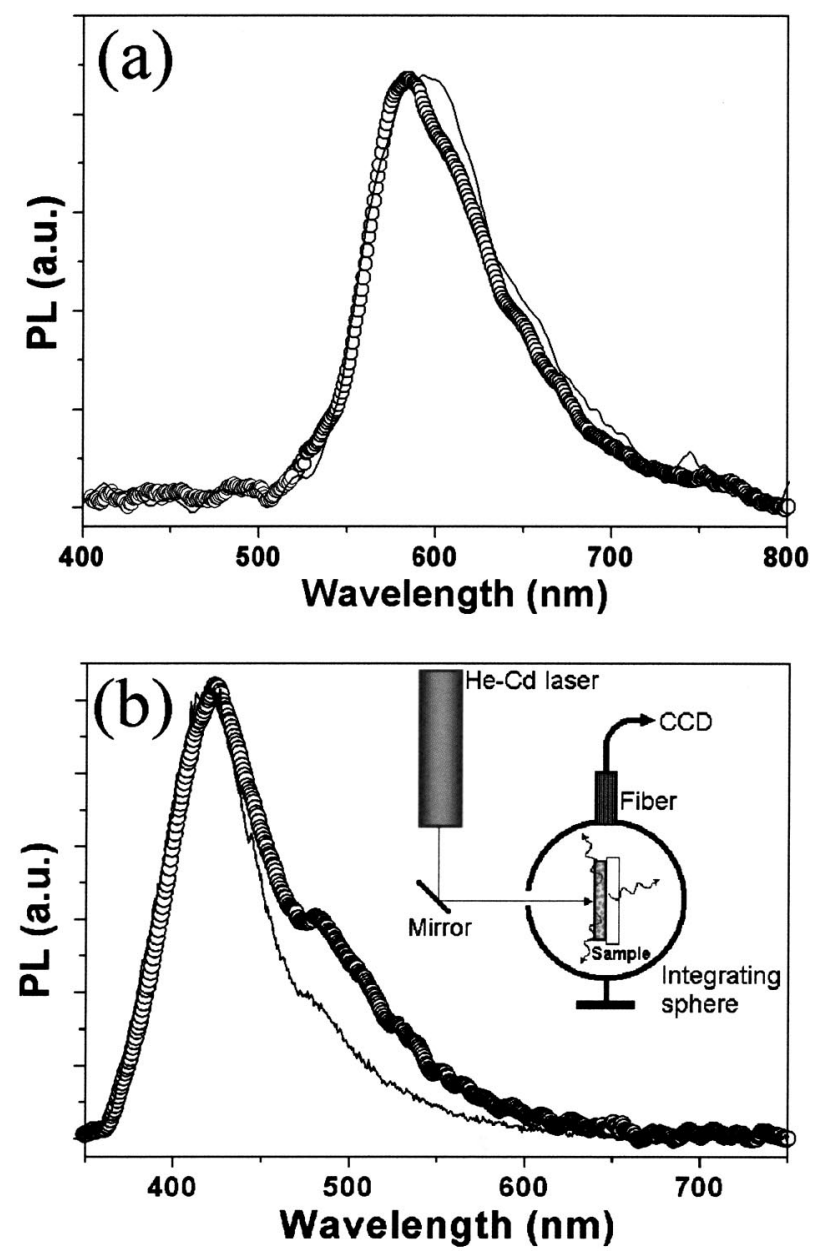

FIG. 5. PL spectra before (circles) and after (line) SM for MEH-PPV (a) and PVK (b), respectively. Inset: Schematic diagram of the experimental setup employed for measurements of the PL absolute quantum efficiency (elements not to scale).

structural relaxation time and, consequently, in viscosity. Consequently, a proper increase of temperature can be exploited to reduce the viscosity of the organic compound, ${ }^{15}$ thus allowing filling of the pattern in a few minutes.

PL spectra of polymer films before and after SM are shown in Fig. 5. The minor differences that can be observed are possible consequences of the lithography process on the films. In order to more thoroughly assess the luminescence properties of patterned conjugated polymers retained, we measured the PL efficiency, $\Phi$, before and after SM. The number of photons emitted per absorbed photon by the untextured and the patterned films was determined by accounting for those photons which are not absorbed by the sample at their first incidence, and are absorbed after successive reflections on the surface of the integrating sphere: ${ }^{10}$

$$
\Phi=\frac{P_{1}-(R+T) P_{2}}{(1-R-T) X_{L}},
$$

where $R$ and $T$ indicate the reflectance and transmittance of the system, respectively. $P_{1}$ and $P_{2}$ are the photoluminescence signals measured with the laser beam incident on the sample and on the integrating sphere, respectively, and $X_{L}$ stands for the excitation signal, measured with the laser incident on the sphere without the sample. The $\Phi$ values did not show relevant degradation, thus demonstrating that hightemperature soft lithography can actually be applied without serious deterioration of the functionality of the target materials. A remarkable increase of $\Phi$ (from $4.9 \%$ to $7.2 \%$ ) was observed in PVK. This can be explained as follows: imprinting of a surface grating with wavelength-scale periodicity can enhance the light emitted towards certain forward directions due to grating-induced Bragg scattering, ${ }^{16,17}$ thus reducing the length covered by the photons and the selfabsorption inside the organic slab. A slight decrease (from $8.0 \%$ to $7.1 \%$ ) of $\Phi$ was instead measured in MEH-PPV: Because the thermal-induced carbonyl substitution in poly(phenylenevinylene) and its derivatives is strongly affected by the process atmosphere, ${ }^{18}$ experiments of SM under higher-vacuum conditions aimed at better preserving the PL quantum yield are currently in progress in our laboratory. In conclusion, we have shown that SM, besides allowing high resolution and fast simple operation, can be applied to light-emitting conjugated polymers without causing degradation. Even though a number of issues for SM need to be investigated further, including its ultimate resolution and the maximum aspect ratio achievable, which could be limited by distortion and the collapse of soft PDMS structures, this technique is undoubtedly very promising for organic-based micro- and nanofabrication.

The authors would like to thank Professor A. Maffezzoli and Engineer G. Raho for DSC measurements, and Dr. F. Della Sala for helpful discussions.

${ }^{1}$ Y. Xia and G. M. Whitesides, Angew. Chem., Int. Ed. 37, 550 (1998).

${ }^{2}$ K. Y. Suh, Y. S. Kim, and H. H. Lee, Adv. Mater. (Weinheim, Ger.) 13, 1386 (2001).

${ }^{3}$ S. Y. Chou, P. R. Krauss, and P. J. Renstrom, Appl. Phys. Lett. 67, 3114 (1995); Science 272, 85 (1996).

${ }^{4}$ K. Y. Suh and Hong H. Lee, Adv. Funct. Mater. 12, 405 (2002).

${ }^{5}$ Y. S. Kim, K. Y. Suh, and Hong H. Lee, Appl. Phys. Lett. 79, 2285 (2001).

${ }^{6}$ L. S. Roman, O. Inganäs, T. Granlund, T. Nyberg, M. Svensson, M. R. Andersson, and J. C. Hummeen, Adv. Mater. (Weinheim, Ger.) 12189 (2000).

${ }^{7}$ G. A. Turnbull, T. F. Krauss, W. L. Barned, and I. D. W. Samuel, Synth. Met. 121, 1757 (2001).

${ }^{8}$ T.-Q. Nguyen, R. C. Kwong, M. E. Thompson, and B. J. Schwartz, Appl. Phys. Lett. 76, 2454 (2000).

${ }^{9}$ J. Kido, K. Hongawa, K. Okuyama, and K. Nagai, Appl. Phys. Lett. 63, 2627 (1993).

${ }^{10}$ N. C. Greenham, I. D. W. Samuel, G. R. Hayes, R. T. Phyllips, Y. A. R. R. Kessener, S. C. Moratti, A. B. Holmes, and R. H. Friend, Chem. Phys. Lett. 241, 89 (1995).

${ }^{11}$ M. Yan, L. J. Rothberg, F. Papadimitrakopoulos, M. E. Galvin, and T. M. Miller, Phys. Rev. Lett. 73, 744 (1994), and references therein.

${ }^{12}$ J. Wang, X. Sun, L. Chen, and S. Y. Chou, Appl. Phys. Lett. 75, 2767 (1999).

${ }^{13}$ D. Myers, Surfaces, Interfaces and Colloids (Wiley-VCH, New York, 1999).

${ }^{14}$ H. Vogel, Phys. Z. 22, 645 (1921); G. S. Fulcher, J. Am. Ceram. Soc. 8, 339 (1925); G. Tamman and W. Hesse, Z. Anorg. Allg. Chem. 156, 245 (1926).

${ }^{15}$ K. L. Ngai and D. J. Plazek, Physical Properties of Polymers Handbook, edited by J. E. Mark (American Institute of Physics, Woodbury, New York, 1996).

${ }^{16}$ B. J. Matterson, J. M. Lupton, A. F. Safonov, M. G. Salt, W. L. Barnes, and I. D. W. Samuel, Adv. Mater. (Weinheim, Ger.) 13, 123 (2001).

${ }^{17}$ G. A. Turnbull, P. Andrews, M. J. Jory, W. L. Barnes, and I. D. W. Samuel, Phys. Rev. B 64, 125122 (2001).

${ }^{18}$ F. Papadimitrakopoulos, K. Konstadinidis, T. M. Miller, R. Opila, E. A. Chandross, and M. E. Galvin, Chem. Mater. 6, 1563 (1994). 